\title{
Non typable-Haemophilus influenzae biofilm formation and acute otitis media
}

\author{
Assaf Mizrahi ${ }^{1}$, Robert Cohen ${ }^{2}$, Emmanuelle Varon ${ }^{3}$, Stephane Bonacorsi ${ }^{4}$, Stephane Bechet ${ }^{2}$, Claire Poyart ${ }^{1}$, \\ Corinne Levy ${ }^{2}$ and Josette Raymond ${ }^{1 *}$
}

\begin{abstract}
Background: Non-typable Haemophilus influenzae (NT-Hi) infection is frequently associated with acute otitis media (AOM) treatment failure, recurrence or chronic otitis media. Persistence of otopathogens in a biofilm-structured community was implicated in these situations. Here, we compared biofilm production by $\mathrm{H}$. influenzae strains obtained by culture of middle ear fluid (MEF) from children with AOM treatment failure and by strains isolated from nasopharyngeal (NP) samples from healthy children or those with AOM (first episode or recurrence). We aimed to evaluate an association of clinical signs and in vitro biofilm formation and establish risk factors of carrying a biofilm-producing strain.
\end{abstract}

Methods: We used a modification of the microtiter plate assay with crystal violet staining to compare biofilm production by $216 \mathrm{H}$. influenzae strains: 41 in MEF from children with AOM treatment failure (group MEF), 43 in NP samples from healthy children (NP group 1), 88 in NP samples from children with a first AOM episode (NP group $2, n=43$ ) or recurrent (NP group 3, $n=45$ ) and 44 in NP samples from children with AOM associated with conjunctivitis (NP group 4).

Results: At all, 106/216 (49\%) H. influenzae strains produced biofilm as did 26/43 (60.5\%) in NP samples from healthy children. Biofilm production in MEF samples and NP samples did not significantly differ (40.5\% vs $60.5 \%$, $55.8 \%, 56.8 \%$ and $31.1 \%$ for NP groups 1, 2, 3 and 4, respectively). On multivariate analysis, only presence of conjunctivitis was significantly associated with low biofilm production $(\mathrm{OR}=0.3, \mathrm{Cl}[0.16-0.60], p=0.001)$. The ampicillin resistance of $\mathrm{H}$. influenzae produced by penicillin-binding protein modification was significantly associated with low biofilm production $(p=0.029)$.

Conclusion: We found no association of biofilm production and AOM treatment failure or recurrence. Biofilm production was low from $\mathrm{H}$. influenzae strains associated with conjunctivitis-otitis syndrome and from strains with modified penicillin-binding protein.

Keywords: Haemophilus influenzae, Biofilm, AOM, Conjunctivitis

\section{Background}

Non-typable H. influenzae (NT-Hi) is a commensal bacterium of the human respiratory tract and can be responsible for non-invasive diseases such as acute otitis media (AOM) and sinusitis. With the expanded use of 7-valent pneumococcal vaccination, Streptococcus pneumoniae and NT-Hi are the two most common bacteria implicated in AOM $[1,2]$. Couloigner et al. recently reported that after the

\footnotetext{
* Correspondence: josette.raymond@cch.aphp.fr

'Université Paris Descartes, Hôpital Cochin, Bactériologie, 27 rue du Faubourg Saint Jacques, 75679 Paris cedex 14, France

Full list of author information is available at the end of the article
}

7-valent Pneumococcal Conjugate Vaccine implementation in France, S. pneumoniae and NT-Hi infection were equally frequent among children with $\mathrm{AOM}$ treatment failure. Indeed, the serotype 19A, not included in the vaccine, was the main S. pneumoniae serotype reported and represented $84.5 \%$ of all serotypes detected [3].

NT- $H i$ is frequently associated with AOM treatment failure, recurrence and otitis media effusion [4,5]. Faden et al. demonstrated that nasopharyngeal (NP) colonization with NT-Hi is an important risk factor for AOM [6]. AOM is more likely to develop in children with than without frequent NP colonization with NT-Hi [6,7]. Kaur et al. 
used multi-locus sequence typing to compare strains isolated from NP and middle ear fluid (MEF) in 34 children during an AOM episode. They found the same sequence type of NT-Hi in 31 (84\%) children, which highlights the close relationship between strains isolated from both sites [8]. Furthermore, because of the pain caused by tympanocentesis, bacteriological samples of MEF are generally not recommended in most guidelines for AOM followed by paediatricians or general practitioners, except in case of treatment failure [8].

Several studies have shown that NT-Hi forms biofilms in vivo and in vitro $[9,10]$. A biofilm is a community of microorganisms adhering to a surface and enclosed in a self-produced extracellular matrix (ECM). This structure protects against external aggression such as the host immune system and antibiotics treatment [11]. Biofilm formation was originally suggested to explain the failure to culture NT-Hi from middle-ear effusions, resistance to antibiotics and pathogenic behavior. Bacterial biofilms are mainly present in chronic infections, such as chronic pulmonary infections caused by Pseudomonas aeruginosa in cystic fibrosis patients or in medical-devicerelated infections mainly due to Staphylococcus aureus or Staphylococcus epidermidis [12,13]. Persistence of NT-Hi in a biofilm-structured community was implicated in the pathogenesis of chronic and recurrent otitis media $[10,11]$. The mechanism seems to be an inefficient clearance of bacteria from the middle ear [14]. However, this hypothesis remains controversial [15].

Here, we aimed to determine whether biofilm production is increased in bacterial strains from children with AOM treatment failure. We compared the in vitro biofilm-forming ability of $H$. influenzae strains in MEF from children with AOM treatment failure and in NP samples from children with a first episode or recurrent AOM with or without conjunctivitis or in NP microbiota from healthy children. In addition, we evaluated a possible association of clinical signs and in vitro biofilm formation and identified risk factors of carrying a $H$. influenzae strain producing biofilm.

\section{Methods}

\section{A) Patients}

After the implementation of PCV7 in France, we conducted two studies in parallel during the same period (May 2007 to April 2009). In the first study, ear, nose and throat specialists obtained MEF samples from children with AOM treatment failure [3]. This study enrolled 143 children (mean age $16.9 \pm 9$ months). The second study examined the NP carriage of $S$. pneumoniae and $H$. influenzae in healthy children and children with AOM [3].

Treatment failure was defined as otorrhea or bulging of the tympanic membrane, together with fever and otalgia (or its equivalent: irritable or ill-tempered child), despite at least $48 \mathrm{hr}$ of antibiotics, or recurring $<4$ days after the end of antimicrobial treatment. Recurrence was defined by the reappearance of AOM signs and symptoms 4 to 30 days after the end of antimicrobial treatment [16]. The definitions of recurrence and failures applied to all patients of all groups.

\section{Establishment of patient groups}

In the first study of 143 children with AOM treatment failure or recurrence, $H$. influenzae was found in MEF cultures from 45 children, which constituted the "MEF group" in this study.

Among the children enrolled in the NP surveillance study, 98 healthy children and 481 children with AOM were carrying $H$. influenzae. Because tympanocentesis is not recommended for first-line treatment of AOM, we used NP samples and stringent criteria for the diagnosis of otitis. We defined 4 NP groups: 1) NP group 1, $H$. influenzae strains in NP samples from 98 healthy children; 2) NP group 2, $H$. influenzae strains in NP samples from 82 children with a first AOM without conjunctivitis; 3) NP group 3, $H$. influenzae strains in NP samples from 57 children with recurrent AOM without conjunctivitis; and 4) NP group 4, H. influenzae strains in NP samples from 342 children with first or recurrent AOM associated with conjunctivitis.

From each of these 4 groups, we randomly selected representative samples of 45 children (one strain per child). For NP group 4 (first or recurrent AOM), we did not have information about history of otitis for 23 children; 13 had previous otitis, and AOM was the first episode for 9 children. Therefore, because of the low number of children in each subgroup (first or recurrent otitis), this group cannot be divided. Co-carriage of H. influenzae and S. pneumoniae in NP samples was considered (Figure 1).

\section{Ethics approval}

The two studies used standardized protocols and common inclusion criteria for AOM and enrolled children of the same age. Furthermore, they involved mostly the same investigators and centralized microbiology laboratories. The protocols were approved by the Saint Germain en Laye Hospital Ethics Committee. Written informed consent was obtained from parents or legal representatives.

\section{B) Methods}

\section{Samples}

Deep NP samples were taken transnasally with use of a flexible, sterile, soft rayon swab tip. After sampling, swabs were immediately inoculated in transport medium (Copan Venturi Transystem, Brescia, Italy), stored at ambient temperature and sent most often during the day 
matrix-assisted laser desorption ionization-time of flight mass spectrometry (MALDI-TOF MS, Bruker Daltonics, Germany) with the database provided by the Haemophilus National Reference Center [20].

Then $H$. influenzae isolates were grown overnight in $\mathrm{BHI}$ broth (Becton Dickinson, Le Pont-De-Claix, France) supplemented with $15 \mathrm{mg} / \mathrm{L}$ nicotinamide adenine dinucleotide and hemin (HTM supplemented, Oxoid, Basingstoke Hampshire, UK). The suspension was washed with sterile phosphate buffered saline and diluted 1:200 in fresh supplemented BHI broth. In total, $200 \mu \mathrm{H}$. influenzae suspension was inoculated into wells of a polystyrene flat-bottomed 96-well microtiter plates (Nunc, Kracker Scientific, Inc, Albany, New York), which were incubated at $37^{\circ} \mathrm{C}$ for $24 \mathrm{hr}$ aerobically with $5 \% \mathrm{CO}_{2}$. The growth of $H$. influenzae was assessed by measuring optical density absorbance at $595 \mathrm{~nm}\left(\mathrm{OD}_{595 \mathrm{~nm}}\right)$. Culture media including unattached bacteria was decanted from wells, and the remaining planktonic $H$. influenzae cells were removed by rinsing with distilled water. The wells were air-dried and adhered bacteria were stained with $0.5 \%$ (w/v) CV solution (Sigma-aldrich, USA) for 15 min. After rinsing with distilled water, bound $\mathrm{CV}$ was released from $H$. influenzae cells by a $20 \%$ acetone$80 \%$ ethanol solution. Thus, biofilm formation could be measured on both bottoms and sides of wells. Biofilm formation was quantified by measuring the absorbance at $\mathrm{OD}_{595 \mathrm{~nm}}$.

A strain of $S$. aureus producing biofilm and a strain of Lactococcus lactis non-producing biofilm were included in each experiment as controls. These strains were kindly provided by the Streptococci national reference center (Cochin Hospital, Paris). The assays were performed in triplicate. For each assay, the BHI broth alone was tested to calculate the biofilm formation index (BFI).

\section{Determination of the cut-off value}

The BFI was determined by use of three different formulas. With the first formula [21], BFI $=\mathrm{AB}-\mathrm{CW}$, where $\mathrm{AB}$ represents the optical density at $595 \mathrm{~nm}\left(\mathrm{OD}_{595 \mathrm{~nm}}\right)$ of a well containing stained attached bacteria and $\mathrm{CW}$, the $\mathrm{OD}_{595 \mathrm{~nm}}$ of the stained control wells containing bacteria-free medium only, here supplemented with $\mathrm{BHI}$. With the second formula [22], BFI $=\mathrm{AB} / \mathrm{CW}$. With the third formula $[23], B F I=(A B-C W) / G$, where $G$ is the $\mathrm{OD}_{595 \mathrm{~nm}}$ of bacterial growth control. The assays were performed in duplicate. Finally, results were studied by terciles to classify biofilm production semiquantitatively in three categories for each formula: strong production $(\mathrm{S})$, moderate production $(\mathrm{M})$ and absence of production $(\mathrm{N})$ according to the cut-off values proposed by Naves et al. [24]. Because Naves et al. [24] showed that biofilm formation was strongly modulated by culture conditions, environmental factors and methodology, we combined the three BFI formulas to overcome above factors.

\section{Statistical analysis}

Double data entry was performed with use of the software $4 \mathrm{D} v 12$. Univariate analysis and multivariate logistic regression models and estimation of odds ratios (ORs) and 95\% confidence intervals [CI] involved use of Stata SE v11.2 (Stata Corp., College Station, TX, USA). The Pearson chi-square test was used to compare groups. Potential risk factors were identified by univariate analysis $(p<0.25)$ and applied in multivariate logistic regression models. Factors considered were history of AOM, conjunctivitis, associated carriage of S. pneumoniae and BLNAR strains. $\mathrm{P}<0.05$ was considered statistically significant.

\section{Results}

Among the 225 selected strains, 9 were not found in the collection of frozen samples and therefore could not be included. So, we analyzed the remaining 216 strains. All strains were identified by MALDI-TOF MS. When the result was not sufficiently discriminative between $H$. influenzae and $H$. haemolyticus, the identification of $H$. influenzae was confirmed by sequencing $\operatorname{sod} A_{\text {int }}, \operatorname{rec} A$ and fucK genes [25].

Among the 171 isolates in NP samples, only 2 (1.1\%) were serotype b $H$. influenzae. All of the 45 strains isolated in MEF were non-typable strains.

Among the 216 strains, 37 produced $\beta$-lactamase (17.1\%), 32 were BLNAR (14.8\%) and 7 showed both mechanisms of resistance (3.2\%).

\section{1) Comparison of the three methods to calculate BFI}

Among the 216 studied strains, 162 (75\%) were classified in the same category of biofilm production (strong, moderate or absence) by the three formulas. For 54 strains, results were discordant. However, in each case, results were concordant with two of the three formulas. Therefore, these 54 strains were classified by agreement in two of the three formulas. Finally, 47/216 (21.7\%) strains of $H$. influenzae were classified as strong producers of biofilm and 59/216 (27.3\%) as moderate producers; 110/216 (51\%) did not produce biofilm. In total, 106 (49\%) strains produced biofilm.

2) Relationship between pathology and biofilm production To describe the effect of biofilm production by pathology, results were combined for two categories: strains producing biofilm (moderate or strong production) and strains not producing biofilm (negative). In the control group of healthy children (NP group 1), $60.5 \%$ H. influenzae strains produced biofilm (Table 1). 
Table 1 Association of pathologic group and biofilm production by isolated $\boldsymbol{H}$. influenzae strains

\begin{tabular}{|c|c|c|c|c|c|c|c|c|}
\hline \multirow[t]{2}{*}{ Groups } & \multicolumn{4}{|c|}{ Level of biofilm production } & \multicolumn{4}{|c|}{ Comparison between groups * $\mathrm{p}$ value OR $[95 \% \mathrm{Cl}]$} \\
\hline & Negative & Moderate & Strong & Moderate + Strong & Reference vs Group 1 & Reference vs Group 2 & Reference vs Group 3 & Reference vs Group 4 \\
\hline Group MEF** $n=41$ & $24(58.5 \%)$ & $8(19.5 \%)$ & $9(22 \%)$ & $17(40.5 \%)$ & 0.083 & 0.19 & 0.16 & 0.32 \\
\hline AOM treatment failure & & & & & $2.16[0.90 ; 5.16]$ & $1.78[0.75 ; 4.24]$ & $1.86[0.79 ; 4.40]$ & $0.64[0.26 ; 1.55]$ \\
\hline NP group $1^{* * *} \mathrm{n}=43$ & $17(39.5 \%)$ & $11(25.6 \%)$ & 15 (34.9\%) & $26(60.5 \%)$ & & 0.66 & 0.73 & 0.006 \\
\hline Control (healthy children) & & & & & & $1.21[0.51 ; 2.86]$ & $1.16[0.49 ; 2.73]$ & $3.39[1.41 ; 8.15]$ \\
\hline NP group $2^{* * *} n=43$ & $19(44.2 \%)$ & $18(41.9 \%)$ & $6(13.9 \%)$ & $24(55.8 \%)$ & & & 0.93 & 0.02 \\
\hline First AOM without conjunctivitis & & & & & & & $0.96[0.41 ; 2.24]$ & $2.8[1.17 ; 6.69]$ \\
\hline NP group $3^{* * *} n=45$ & 19 (43.2\%) & $13(29.5 \%)$ & $12(27.3 \%)$ & $25(56.8 \%)$ & & & & 0.015 \\
\hline Recurrent $\mathrm{AOM}$ without conjunctivitis & & & & & & & & $0.34[0.14 ; 0.82]$ \\
\hline NP group $4^{* * *} n=44$ & 31 (68.9\%) & $9(20 \%)$ & $5(11.1 \%)$ & $14(31.1 .5 \%)$ & & & & \\
\hline AOM with conjunctivitis & & & & & & & & \\
\hline
\end{tabular}

OR, odds ratio; $95 \% \mathrm{Cl}, 95 \%$ confidence interval.

*Risk for carrying (moderate + strong) an $\mathrm{H}$. influenzae strain producing biofilm.

**Strains isolated from MEF.

***Strains isolated from the nasopharynx. 
Considering the influence of biofilm production on AOM recurrence, we found no difference between strains in NP during a first AOM episode or during recurrent $\mathrm{AOM}$ in the absence of conjunctivitis (NP group 2 vs NP group 3, 55.8\% vs 56.8\%, respectively, $p=0.93, \mathrm{OR}=0.96$ [95\% CI, 0.41; 2.24]) (Table 1).

We found no significant difference in biofilm production from strains in MEF (children with AOM treatment failure) and strains in NP samples (NP groups 1, 2, 3 and 4$)$ : 40.5\% vs 60.5\% ( $p=0.083$, OR = $2.16[0.90 ; 5.16]), 55.8 \%(p=0.19,1.78[0.75 ; 4.24])$, $56.8 \%(p=0.16,1.86[0.79 ; 4.40])$ and $31.1 \%(p=0.32$, $0.64[0.26 ; 1.55])$, respectively.

However, $H$. influenzae strains in NP samples from children with both AOM and conjunctivitis (NP group 4) produced significantly less biofilm (68.9\% non-producer) than strains from NP groups 1,2 and $3(p=0.006$ and $\mathrm{OR}=3.39[1.41 ; 8.15], p=0.0 ; 2.8$ $[1.17 ; 6.69], p=0.015$; and $0.34[0.14 ; 0.82]$, respectively). Biofilm production did not significantly differ between strains in MEF and in NP samples from children with AOM and conjunctivitis. For most of the comparisons, the number of patients was too small and the differences between groups too low to demonstrate significant differences.

Finally, biofilm production did not significantly differ between NP group 1 strains (healthy children, $60.5 \%)$ and those from children with a first or recurrent AOM without conjunctivitis, NP groups 2 $(55.8 \%, p=0.66, \mathrm{OR}=1.21[0.51 ; 2.86])$ and $3(56.8 \%$, $p=0.73,1.16[0.49 ; 2.73)$.

3) Risk factors for carriage of $H$. influenzae producing biofilm

We examined associations between risk factors for $H$. influenzae carriage and biofilm production by univariate analysis. Age less or greater than 12 months old as well as type of daycare (daycare center, home or nurse) were not associated with carriage of a strain producing biofilm ( $p=0.36$ and 0.61 , respectively) (Table 2).

$H$. influenzae was often found with $S$. pneumoniae (44.9\% of cases). Although $H$. influenzae produced more biofilm with than without S. pneumoniae cohabitation $(\mathrm{OR}=1.6 \mathrm{CI}[0.94 ; 2.78])$, the difference was not significant $(55.7 \%$ with vs $43.7 \%$ without cohabitation, $p=0.08)$. Antibiotic treatment, amoxicillin or third-generation cephalosporin taken in the previous 3 months was not a risk factor for carrying an $H$. influenzae strain producing biofilm, $p=0.71$ (Table 2).

Two factors were significantly associated with low biofilm production. The first was ampicillin resistance of $H$. influenzae by modification of the penicillin-binding protein (PBP) (BLNAR strains) $(31.2 \%$ producing biofilm by ampicillin-resistant strains $v s \quad 52.2 \%$ by ampicillin-susceptible strains, $p=0.029 ; \mathrm{OR}=2.4 \mathrm{CI}$ [1.08; 5.35]) (Table 2). The resistance to ampicillin mediated by $\beta$-lactamase did not affect biofilm production. The second risk factor associated with decreased biofilm production was the presence of conjunctivitis-otitis syndrome. Indeed, strains causing otitis and conjunctivitis produced significantly less biofilm than those from children with otitis without conjunctivitis: $27.8 \%$ vs $55.6 \%, p<0.001(\mathrm{OR}=3.3$, CI [1.66; 6.38]).

\section{4) Multivariate analysis}

We examined predictors of low biofilm production by $H$. influenzae strains by multivariate analysis of past history of AOM, presence of conjunctivitis, co-colonization with $S$. pneumoniae and PBP modification. Only presence of conjunctivitis was significantly associated with low biofilm production $(p=0.001, \mathrm{OR}=0.3$, CI [0.16-0.60]). The OR was not adjusted.

\section{Discussion}

In this study, we examined whether $H$. influenzae strains in MEF from children with AOM were high biofilm producers, causing AOM treatment failure. We found conjunctivitis-otitis syndrome significantly associated with low biofilm production by $H$. influenzae strains $(27.8 \%$ production with the syndrome vs $55.6 \%$ without). This association was not previously described. In the absence of conjunctivitis, biofilm production did not significantly differ between strains from children with a first, cured AOM and those with recurrent AOM.

One limitation of the study is the small number of patients in each group which does not allow for determining a significant difference in biofilm production by $H$. influenzae between strains from healthy controls and the MEF group (children with AOM treatment failure).

A second limitation is to compare strains from the MEF and NP samples. Some argue that strains in NP samples are not responsible for AOM. In a prospective study, Bingen et al. showed by pulsed-field electrophoresis that in conjunctivitis-otitis syndrome, $H$. influenzae strains from MEF and conjunctivitis were identical for the same child [26]. These data are reinforced by the fact that NT-Hi strains were not clonal and constituted a considerably diverse population. Van Dongen et al. [27], in a systematic review of the literature on the concordance between strains isolated from nasopharyngeal and MEF samples, found that $H$. influenzae strains were concordant in $80 \%$ of the cases.

AOM is the most common bacterial infection in children with $H$. influenzae and S. pneumoniae as the major causative agents. H. influenzae is often found in samples from children with recurrent AOM or AOM 
Table 2 Risk factors for carriage of $\boldsymbol{H}$. influenzae associated with biofilm production

\begin{tabular}{|c|c|c|c|c|c|}
\hline & No. of strains (\%) & Biofilm non-producer* $(n=110)$ & Biofilm producer** $(n=106)$ & P value & OR \\
\hline \multicolumn{6}{|l|}{ Age: $n=216$} \\
\hline$<12$ months & $79(36.6 \%)$ & $37(46.8 \%)$ & $42(53.2 \%)$ & \multirow[t]{2}{*}{0.361} & \\
\hline$>12$ months & $137(63.4 \%)$ & $73(53.3 \%)$ & $64(46.72 \%)$ & & \\
\hline \multicolumn{6}{|c|}{ Types of daycare: $n=216$} \\
\hline Daycare center & $117(54.2 \%)$ & $61(52.1 \%)$ & $56(47.9 \%)$ & \multirow[t]{3}{*}{0.616} & \\
\hline Home & $49(22.7 \%)$ & $22(44.9 \%)$ & $27(55.1 \%)$ & & \\
\hline Nurse & $50(23.1 \%)$ & $27(54 \%)$ & $23(46 \%)$ & & \\
\hline \multicolumn{6}{|c|}{ Association with Streptococcus pneumoniae: $n=216$} \\
\hline Yes & $97(44.9 \%)$ & $43(44.3 \%)$ & $54(55.7 \%)$ & \multirow[t]{2}{*}{0.08} & \\
\hline No & $119(55.1 \%)$ & $67(56.3 \%)$ & $52(43.7 \%)$ & & \\
\hline \multicolumn{6}{|c|}{$\beta$-lactamase production: $n=216$} \\
\hline Yes & $37(17.1 \%)$ & $21(56.80 \%)$ & $16(43.20 \%)$ & \multirow[t]{2}{*}{0.436} & \\
\hline No & $179(82.9 \%)$ & $89(49.72 \%)$ & $90(50.28 \%)$ & & \\
\hline \multicolumn{6}{|c|}{ Penicillin-binding protein modification: resistance to amoxicillin: $\mathrm{n}=\mathbf{2 1 6}$} \\
\hline Yes & $32(14.8 \%)$ & $22(68.75 \%)$ & $10(31.25 \%)$ & \multirow[t]{2}{*}{0.029} & \multirow[t]{2}{*}{$2.4[1.08 ; 5.35]$} \\
\hline No & $184(85.2 \%)$ & $88(47.83 \%)$ & $96(52.17 \%)$ & & \\
\hline \multicolumn{6}{|c|}{ History of AOM: $n=172$} \\
\hline Yes & $104(60.5 \%)$ & $54(51.9 \%)$ & $50(48.1 \%)$ & \multirow[t]{2}{*}{0.168} & \\
\hline No & $68(39.5 \%)$ & $28(41.2 \%)$ & $40(58.8 \%)$ & & \\
\hline \multicolumn{6}{|c|}{ Associated conjunctivitis: $n=214$} \\
\hline Yes & $54(25.2 \%)$ & $39(72.2 \%)$ & $15(27.8 \%)$ & \multirow[t]{2}{*}{$<0.001$} & \multirow[t]{2}{*}{$3.3[1.66 ; 6.38]$} \\
\hline No & $160(74.8 \%)$ & $71(44.4 \%)$ & $89(55.6 \%)$ & & \\
\hline \multicolumn{6}{|c|}{ Previous antimicrobial therapy ( $<3$ months): $n=210$} \\
\hline None & $91(43.3 \%)$ & $43(47.2 \%)$ & $48(52.8 \%)$ & \multirow[t]{3}{*}{0.716} & \\
\hline Amoxicillin & $70(33.3 \%)$ & $37(52.9 \%)$ & $33(47.1 \%)$ & & \\
\hline $3 G C^{* * *}$ & 49 (23.4\%) & $26(53.1 \%)$ & $23(46.9 \%)$ & & \\
\hline \multicolumn{6}{|c|}{ Pneumococcal vaccination: $\mathrm{n}=\mathbf{2 1 6}$} \\
\hline Yes & $211(97.7 \%)$ & $106(50.2 \%)$ & $105(49.8 \%)$ & \multirow[t]{2}{*}{0.188} & \\
\hline No & $5(2.3 \%)$ & $4(80.0 \%)$ & $1(20.0 \%)$ & & \\
\hline
\end{tabular}

*No. of strains not producing biofilm.

**No. of strains producing biofilm.

***3GC: third-generation cephalosporins.

treatment failure [4]. A recent hypothesis was the ability of $H$. influenzae to produce biofilm and thus escape the antimicrobial treatment [11]. Bacterial growth in a selfproduced ECM, preventing the action of antibiotics and an efficient host immune system, can explain the chronic nature of various infections. However, this hypothesis is still controversial [15]. Recently, Swords et al. [28] discounted the relevance of NT-Hi biofilms in disease. Langereis et al. [29] suggested that NT-Hi can lie in biofilm during both colonization and infection. The definition of a biofilm is still being discussed. Toretta et al. [30] reported greater biofilm production from NT-Hi from children with AOM than from healthy children. However, this study included only 12 AOM strains and only one healthy control strain.

We found significantly low biofilm production in strains with than without modified PBP $(\mathrm{OR}=2.4$ (CI $[1.08 ; 5.35])$. One hypothesis for this phenomenon is that resistance to amoxicillin and amoxicillin/clavulanic acid may promote the planktonic state. As well, biofilm production could be considered a real mechanism of resistance, and ß-lactamase production could obviate any resistance mechanism. These data need to be confirmed and explored in further studies.

The ECM secreted by $H$. influenzae is composed of lipo-oligosaccharides, proteases and adhesins but not a 
specific biofilm protein [31]. We studied biofilm production by $H$. influenzae stains with the "biofilm ring test" (Biofilm Control, Saint Beauzire, France) [32]. In the absence of a standardized method for $H$. influenzae (mainly due to the broth medium used and the need for $5 \% \mathrm{CO}_{2}$ atmosphere), non-reproducible results were obtained. Therefore, we evaluated biofilm production of $H$. influenzae by the modified microtiter plate assay with CV staining [18]. However, this method still has the drawback of possibly removing the established biofilm when wells are washed. As well, CV as a cationic dye can stain a biofilm complex structure but also a group of living and dead bacteria. A limitation of our study is the lack of direct evaluation of biofilm on biological samples with alternative methods such as confocal microscopy after live/ dead staining [33,34]. This method cannot be used with swabs and cannot be applied retrospectively. Furthermore, although swabs were in a transport medium, we cannot exclude that the delay in bacterial culture influenced the results. Therefore, we used three formulas to calculate biofilm production, and quantifying bacterial biofilm.

Rayner et al. hypothesized that $H$. influenzae could not be isolated from MEF cultures because the biofilm production disallowed cultivation [34]. AOM bacterial etiology was suspected because bacterial genetic material was found by RT-PCR. This hypothesis can explain the lower rate of biofilm-producing strains in the MEF group (treatment failure). Indeed, in this group, a double population may exist and the isolated strain was not the one producing biofilm; the responsive one may have been inside a biofilm structure and could not grow on agar plates.

In another study of 62 children with AOM, 84.3\% of strains produced biofilm [33]. Nevertheless, this study lacked a control group. Clinical follow-up showed no significant difference in biofilm production between strains isolated from children with cured AOM and from patients with AOM treatment failure after 14 days of appropriate antibiotic therapy (78.6\% vs 90\%).

The co-localization of NT-Hi and S. pneumoniae suggests a synergistic interaction between the two organisms. To explain AOM treatment failure in the chinchilla middle ear, Weimer et al. suggested that $H$. influenzae and S. pneumoniae cohabitation may promote biofilm production of S. pneumoniae [35]. However, Tikhomirova A. et al. [36] questioned whether the relation between $H$. influenzae and S. pneumoniae is competitive or cooperative. We found no difference in biofilm production with H.influenzae alone or combined with S. pneumoniae.

Slinger et al. showed that MICs of $H$. influenzae for various antibiotics (amoxicillin, amoxicillin/clavulanic acid, ciprofloxacin) were higher when bacteria are living in the biofilm than in the planktonic state [37]. As well, Starner et al. showed that a low concentration of various antibiotics stimulated biofilm production by $H$. influenzae [38]. We determined whether prior administration of antibiotics was a risk factor of carrying a biofilm-producing strain. Indeed, according to the biofilm definition, bacteria living in an ECM resist the action of antibiotics. Conversely, Ehrlich et al. showed that after inoculation of $H$. influenzae into the middle ear of chinchillas, strains produced an ECM whose amount increased with time [39]. Then, after an administration of antibiotics $96 \mathrm{hr}$, bacterial biofilm disappeared, whereas, by definition, it was expected to be resistant to antibiotics. We found no significant difference in biofilm production among strains from children with and without antibiotics treatment (amoxicillin/clavulanic acid or 3GC) in the previous 3 months. We did not confirm that prior administration of antibiotics affected biofilm production by $H$. influenzae.

Finally, we observed two kinds of strains: 1) strains with a high production of biofilm (55.8\% to 60.5\%) in NP samples from healthy children and from AOM children without conjunctivitis and 2) strains with a lower production of biofilm (40.5\% and 31.15\%) in MEF or NP samples from children with AOM and conjunctivitis, respectively.

Recently, Sanchez et al. [40] examined whether $S$. pneumoniae in biofilms was virulent and contributed to invasive pneumococcal disease (IPD) development. The authors suggested that biofilms did not directly contribute to development of IPD and may instead confer a quiescent mode of growth during colonization. They challenged mice with equal colony-forming units of biofilm and planktonic pneumococci and determined that biofilm bacteria production was highly attenuated in invasive disease but not NP colonization.

Finally, we observed two kinds of strains: 1) strains with a high production of biofilm (55.8\% to 60.5\%) in NP samples from healthy children and from AOM children without conjunctivitis and 2) strains with a lower production of biofilm (40.5\% and 31.15\%) in MEF or NP samples from children with AOM and conjunctivitis, respectively.

\section{Conclusions}

This study emphasizes the frequency of biofilm production by $H$. influenzae and also the complexity of the phenomenon, because $49 \%$ of the strains produced biofilm. We did not find increased biofilm production in strains isolated from children with AOM treatment failure or recurrent AOM. H. influenzae involved in the conjunctivitis-otitis syndrome showed significantly low biofilm production. We observed the same low production in strains with PBP modification, conferring resistance to ampicillin, as compared with susceptible strains. These observations require study of a larger number of strains to confirm these results and explore other 
directions. To our knowledge, this is the first study evaluating the biofilm production by $H$. influenzae in NP samples from healthy children. With $49 \%$ of the strains producing biofilm, growing inside a biofilm may constitute a natural mode of growth for the bacteria, especially in the upper airways of children.

\section{Competing interests}

The authors declare that they have no competing interests.

\section{Authors' contributions}

AM performed experiments and wrote the manuscript. JR led the study and write the manuscript. SB and $\mathrm{CL}$ designed the study and performed the statistical analysis. SB and EV provided the strains. RC write the manuscript. All authors read and approved the final manuscript.

\section{Author details}

'Université Paris Descartes, Hôpital Cochin, Bactériologie, 27 rue du Faubourg Saint Jacques, 75679 Paris cedex 14, France. ${ }^{2}$ ACTIV, Saint Maur des Fossés, Paris, France. ${ }^{3}$ Université Paris Descartes, Hôpital Georges Pompidou, Bactériologie, Paris, France. ${ }^{4}$ Université Diderot, Hôpital Robert Debré, Bactériologie, Paris, France.

Received: 21 February 2014 Accepted: 12 July 2014

Published: 19 July 2014

\section{References}

1. Casey JR, Pichichero ME: Changes in frequency and pathogens causing acute otitis media in 1995-2003. Pediatr Infect Dis J 2004, 23(9):824-828.

2. Block SL, Hedrick J, Harrison CJ, Tyler R, Smith A, Findlay R, Keegan E: Community-wide vaccination with the heptavalent pneumococcal conjugate significantly alters the microbiology of acute otitis media. Pediatr Infect Dis J 2004, 23(9):829-833.

3. Couloigner V, Levy C, François M, Bidet P, Hausdorff WP, Pascal T, Boucherat M, Bingen E, Mariani P, Pierrot S, Bille E, Carbonnelle E, Varon E, Cohen R: Pathogens implicated in acute otitis media failures after 7-valent pneumococcal conjugate vaccine implementation in France: distribution, serotypes, and resistance levels. Pediatr Infect Dis J 2012, 31(2):154-158.

4. Schwartz RH, Rodriguez WJ, Khan WN: Persistent purulent otitis media. Clin Pediatr (Phila) 1981, 20(7):445-447.

5. Barkai G, Leibovitz E, Givon-Lavi N, Dagan R: Potential contribution by nontypable Haemophilus influenzae in protracted and recurrent acute otitis media. Pediatr Infect Dis J 2009, 28(6):466-471.

6. Faden H, Duffy L, Wasielewski R, Wolf J, Krystofik D, Tung Y: Relationship between nasopharyngeal colonization and the development of otitis media in children: Tonawanda/Williamsville Pediatrics. J Infect Dis 1997, 175(6):1440-1445

7. Cohen R, Levy C, Bingen E, Bechet S, Derkx V, Werner A, Koskas M, Varon E: [Nasopharyngeal carriage of children 6 to 60 months during the implementation of the 13-valent pneumococcal conjugate vaccine]. Arch Pediatr 2012, 19(10):1132-1139.

8. Kaur R, Chang A, Xu Q, Casey JR, Pichichero M: Phylogenetic relatedness and diversity of non-typable Haemophilus influenzae in the nasopharynx and middle ear fluid of children with acute otitis media. J Med Microbiol 2011, 60(Pt 12):1841-1848.

9. Murphy TF, Kirkham C: Biofilm formation by nontypeable Haemophilus influenzae: strain variability, outer membrane antigen expression and role of pili. BMC Microbiol 2002, 2:7

10. Hall-Stoodley L, Hu FZ, Gieseke A, Nistico L, Nguyen D, Hayes J, Forbes M, Greenberg DP, Dice B, Burrows A, Wackym PA, Stoodley P, Post JC, Ehrlich $G D$, Kerschner JE: Direct detection of bacterial biofilms on the middle-ear mucosa of children with chronic otitis media. JAMA 2006, 296(2):202-211.

11. Costerton JW, Stewart PS, Greenberg EP: Bacterial biofilms: a common cause of persistent infections. Science 1999, 284(5418):1318-1322.

12. Otto M: Staphylococcal Infections: mechanisms of biofilm maturation and detachment as critical determinants of pathogenicity. Annu Rev Med 2013, 64:175-188.

13. Fernández-Olmos A, García-Castillo M, Maiz L, Lamas A, Baquero F, Cantón R: In vitro prevention of Pseudomonas aeruginosa early biofilm formation with antibiotics used in cystic fibrosis patients. Int J Antimicrob Agents 2012, 40(2):173-176

14. Hotomi M, Yamanaka N, Shimada J, Ikeda Y, Faden H: Factors associated with clinical outcomes in acute otitis media. Ann Otol Rhinol Lanyngol 2004, 113(10):846-852.

15. Moxon ER, Sweetman WA, Deadman ME, Ferguson DJP, Hood DW: Haemophilus influenzae biofilms: hypothesis or fact? Trends Microbiol 2008, 16(3):95-100

16. Agence Française de sécurité sanitaire des produits de santé: Antibiotic therapy in current practice in upper respiratory tract infections in adults and children. arguments. Méd mal infect 2005, 35:635-694.

17. CASFM: Recommendations. 2012. Available at: www.sfm-microbiologie.org.

18. O'Toole GA, Kolter R: Initiation of biofilm formation in Pseudomonas fluorescens WCS365 proceeds via multiple, convergent signalling pathways: a genetic analysis. Mol Microbiol 1998, 28(3):449-461.

19. Sutherland I: Biofilm exopolysaccharides: a strong and sticky framework. Microbiology 2001, 147:3-9.

20. Spinali S: Pasteurellaceae Identification by MALDI-TOF Mass Spectrometry. MD Thesis. 2011.

21. Kadurugamuwa JL, Sin L, Albert E, Yu J, Francis K, DeBoer M, Rubin M, Bellinger-Kawahara C, Parr Jr TR Jr, Contag PR: Direct continuous method for monitoring biofilm infection in a mouse model. Infect Immun 2003, 71(2):882-890

22. Soto SM, Smithson A, Horcajada JP, Martinez JA, Mensa JP, Vila J: Implication of biofilm formation in the persistence of urinary tract infection caused by uropathogenic Escherichia coli. Clin Microbiol Infect 2006, 12(10):1034-1036.

23. Niu C, Gilbert ES: Colorimetric method for identifying plant essential oil components that affect biofilm formation and structure. Appl Environ Microbiol 2004, 70(12):6951-6956.

24. Naves P, del Prado G, Huelves L, Gracia M, Ruiz V, Blanco J, RodríguezCerrato V, Ponte MC, Soriano F: Measurement of biofilm formation by clinical isolates of Escherichia coli is method-dependent. J Appl Microbiol 2008, 105(2):585-590.

25. Norskov-Lauritsen N, Overballe MD, Kilian M: Delineation of the species Haemophilus influenzae by phenotype, multilocus sequence phylogeny, and detection of marker genes. J Bacteriol 2009, 191(3):822-831.

26. Bingen $E$, Cohen R, Jourenkova N, Gehanno P: Epidemiologic study of conjunctivitis-otitis syndrome. Pediatr Infect Dis J 2005, 24(8):731-732.

27. van Dongen TM, van der Heijden GJ, van Zon A, Bogaert D, Sanders EA, Schilder AG: Evaluation of concordance between the microorganisms detected in the nasopharynx and middle ear of children with otitis media. Pediatr Infect Dis J 2013, 32(5):549-552.

28. Swords WE: Nontypeable Haemophilus influenzae biofilms: role in chronic airway infections. Front Cell Infect Microbiol 2012, 2:97.

29. Langereis JD, Hermans PW: Novel concepts in nontypeable Haemophilus influenzae biofilm formation. FEMS Microbiol Lett 2013, 346(2):81-89.

30. Torretta S, Marchisio P, Drago L, Baggi E, De Vecchi E, Garavello W, Nazzari E, Pignataro L, Esposito S: Nasopharyngeal biofilm-producing otopathogens in children with nonsevere recurrent acute otitis media. Otolanyngol Head Neck Surg 2012, 146(6):991-996

31. Webster P, Wu S, Gomez G, Apicella M, Plaut AG, St Geme JW III: Distribution of bacterial proteins in biofilms formed by non-typeable Haemophilus influenzae. J Histochem Cytochem 2006, 54(7):829-842.

32. Chavant $P$, Gaillard-Martinie B, Talon R, Hébraud M, Bernardi T: A new device for rapid evaluation of biofilm formation potential by bacteria. J Microbiol Methods 2007, 68(3):605-612.

33. Moriyama S, Hotomi M, Shimada J, Billal DS, Fujihara K, Yamanaka N: Formation of biofilm by Haemophilus influenzae isolated from pediatric intractable otitis media. Auris Nasus Larynx 2009, 36(5):525-531.

34. Rayner MG, Zhang Y, Gorry MC, Chen Y, Post JC, Ehrlich GD: Evidence of bacterial metabolic activity in culture-negative otitis media with effusion. JAMA 1998, 279(4):296-299.

35. Weimer KE, Armbruster CE, Juneau RA, Hong W, Pang B, Swords WE: Coinfection with Haemophilus influenzae promotes pneumococcal biofilm formation during experimental otitis media and impedes the progression of pneumococcal disease. J Infect Dis 2010, 202(7):1068-1075.

36. Tikhomirova A, Kidd SP: Haemophilus influenzae and Streptococcus pneumoniae: living together in a biofilm. Pathog Dis 2013, 69(2):114-126.

37. Slinger R, Chan F, Ferris W, Yeung SW, St Denis M, Gaboury I, Aaron SD: Multiple combination antibiotic susceptibility testing of nontypeable 
Haemophilus influenzae biofilms. Diagn Microbiol Infect Dis 2006, 56(3):247-253.

38. Starner TD, Zhang N, Kim G, Apicella MA, McCray PB Jr: Haemophilus influenzae forms biofilms on airway epithelia: im plications in cystic fibrosis. Am J Respir Crit Care Med 2006, 174(2):213-220.

39. Ehrlich GD, Veeh R, Wang X, Costerton JW, Hayes JD, Hu FZ, Daigle BJ, Ehrlich MD, Post JC: Mucosal biofilm formation on middle-ear mucosa in the chinchilla model of otitis media. JAMA 2002, 287(13):1710-175.

40. Sanchez CJ, Kumar N, Lizcano A, Shivshankar P, Dunning Hotopp JC, Jorgensen $\mathrm{JH}$, Tettelin $\mathrm{H}$, Orihuela CJ: Streptococcus pneumoniae in biofilms are unable to cause invasive disease due to altered virulence determinant production. PLoS One 2011, 6(12):e28738.

doi:10.1186/1471-2334-14-400

Cite this article as: Mizrahi et al:: Non typable-Haemophilus influenzae biofilm formation and acute otitis media. BMC Infectious Diseases 2014 14:400.

\section{Submit your next manuscript to BioMed Central and take full advantage of:}

- Convenient online submission

- Thorough peer review

- No space constraints or color figure charges

- Immediate publication on acceptance

- Inclusion in PubMed, CAS, Scopus and Google Scholar

- Research which is freely available for redistribution 